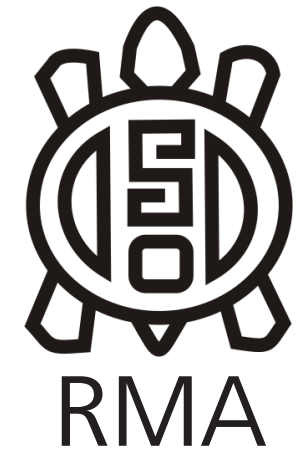

Antropología Biológica

\section{Recuperación, curación y estudios bioantropológicos de la Colección Frengüelli-Brunet}

\author{
Recovery, curation and bioanthropological studies of Frengüelli- \\ Brunet Collection
}

Ana Gabriela Millán*, Nadia Mohamed** y Silvia Dahinten***

*Instituto de Diversidad y Evolución Austral, CONICET, Argentina.

E-mail: millan@cenpat-conicet.gob.ar

**Secretaría de Cultura de la Provincia del Chubut, Argentina. E-mail: nnmohamed@gmail.com

***Facultad de Ciencias. Naturales, Universidad Nacional de la Patagonia San Juan Bosco, Sede

Puerto Madryn. Instituto de Diversidad y Evolución Austral, CONICET, Argentina.

E-mail: dahinten@cenpat-conicet.gob.ar

\title{
Resumen
}

El objetivo del presente trabajo es dar a conocer la Colección Bioantropológica Frengüelli-Brunet, constituida por Rodolfo F. J. Brunet (1926-1996), naturalista y aficionado a la arqueología de la ciudad de Puerto Madryn, que se integra por restos humanos procedentes principalmente de la provincia del Chubut. En la actualidad esta colección se encuentra bajo el amparo de la Ley Provincial XI No 11 sobre "Régimen de las Ruinas y Yacimientos Arqueológicos, Antropológicos y Paleontológicos". La colección ingresó al repositorio Bioantropológico del IDEAUS-CONICET a solicitud de la Secretaría de Cultura del Chubut. Se realizó el proceso de curaduría y protección basado en las normas éticas vigentes. Se determinó información bioantropológica básica: cantidad de individuos, determinación del sexo y estimación de la edad. Se efectuó la revisión de documentación asociada a la colección, a partir de la cual se obtuvo información sobre los sitios de hallazgo Si bien esta colección se conforma a partir de la recuperación asistemática de los esqueletos, sin datos cronológicos y de contexto, la mayoría provienen de la costa central y la región centro oeste de Chubut, cuya información previa es escasa. En este sentido su puesta en valor permitirá sumar aportes sobre las poblaciones originarias de la región.

Palabras Clave: Restos óseos humanos; Recuperación; Colección; Chubut.

\begin{abstract}
The main objective of the present study is to provide information about the Bioanthropological Frengüelli-Brunet collection, built by Rodolfo Brunet (1926-1996), an amateur naturalist from Puerto Madryn city, which is composed of human remains mainly from Chubut province. Currently, this collection is under protection by the Provincial Law XI No 11 which refers to "Archaeological, Anthropological and Paleontological Ruins and Sites Regime". At the request of Chubut Secretary of Culture (Secretaría de Cultura del Chubut), the collection was donated to the IDEAUSCONICET bioanthropological repository. A stringent process of curatorial and protection was performed following current ethical standards. Basic information was determined such as number of individuals, biological sex and age estimation. Information about the place of origin of the collection was obtained through review of documentation associated to the remains. Despite the lack of chronological and archaeological context information due to the unsystematic archaeological rescues, it is worth it to mention that the vast majority of the skeletons in the collection stem from the Central cost and the Central-West Chubut province, a region with scarce archaeological information. This highlights the importance of studying this collection as it will provide valuable knowledge about the original populations that habited the region.
\end{abstract}

Keywords: Human skeletal remains; Recovery; Collection; Chubut. 


\section{Introducción}

Los restos arqueológicos y bioarqueológicos despiertan el interés de las personas, entre ellos aficionados sin la formación profesional adecuada, quienes en general tienden a tomar piezas de toda naturaleza para formar colecciones privadas. Un ejemplo de ello fue Rodolfo F. J. Brunet, naturalista aficionado, que entre los años 1970 a 1990 realizó numerosos recorridos en terreno patagónico, extrayendo restos malacológicos, paleontológicos, arqueológicos y bioantropológicos. De esta forma los materiales recolectados constituyeron distintas colecciones que integraron parte del patrimonio de la Fundación Joaquín Frengüelli, fundada por Brunet el 10 de junio de 1997 e inscripta en la Secretaría de Cultura del Chubut bajo el expediente N¹62-GB-97. Uno de los objetivos de la Fundación Frengüelli y de su creador fue la de generar conocimiento y transmitirlo a la comunidad. En este contexto, el Sr. Brunet fundó el Club de Ciencias Municipal, entidad que acerca a los niños y adolescentes de Puerto Madryn a las ciencias naturales, escribió también guías educativas de malacología y de paleontología (Brunet 1983 y 1995) y donó diversos materiales al Museo Provincial de Ciencias Naturales y Oceanográfico. Tal vez su proyecto más ambicioso fue la construcción de un museo para albergar sus colecciones. Si bien la Corporación de Fomento de CHUBUT S.A. (CORFO) donó a la fundación un terreno para este fin y aun teniendo los planos aprobados, nunca se consiguieron los fondos para su construcción.

Al morir el Sr. Brunet y luego su esposa, las colecciones quedaron depositadas en una casa pequeña durante algún tiempo, hasta que sus herederos solicitaron a la Secretaría de Cultura la reubicación de los materiales. En febrero de 2017 dicha secretaría solicitó al Instituto de Diversidad y Evolución Austral (IDEAUS-CONICET) la custodia de los materiales arqueológicos y bioantropológicos. El objetivo del presente trabajo es dar a conocer las tareas de recuperación, identificación y conservación realizadas a partir de los restos humanos que integraron la sección bioantropológica de la colección de Brunet, denominada ahora Colección Bioantropológica Frengüelli-Brunet, y cogestionada actualmente por la Secretaría de Cultura de Chubut y el IDEAUS-CONICET.

\section{Organización de la Colección Frengüelli-Brunet y Metodología de trabajo.}

\section{Obtención de los materiales}

Al momento de la entrega de la colección a la secretaría de Cultura por parte de los herederos del Sr. Brunet, el equipo de bioantropología del IDEAUS-CONICET efectuó una primera evaluación del lugar de depósito de los materiales, respondiendo a la solicitud de la Secretaría de Cultura, ente regulador de la Ley Provincial XI “Régimen de las ruinas y yacimientos arqueológicos, antropológicos y paleontológicos". Los restos se encontraban en un pequeño departamento de aproximadamente $15 \mathrm{mts} \mathrm{s}^{2}$ que estaba separado de la vivienda familiar, en estado de deterioro edilicio, repleto de cajas de distinto tamaño acopiadas sobre el suelo, en vitrinas y sobre estanterías (Figura 1), sin orden aparente y sin ningún control de plagas y limpieza.

En primera instancia se realizó un relevamiento del material in situ. Se localizaron y contabilizaron las cajas que contenían restos óseos humanos, utilizando el número de registro que figuraba en el Libro de Actas de la Fundación Joaquín Frengüelli. Se revisó el contenido de cada caja, se realizó un inventario preliminar. Posteriormente se trasladó el material desde la vivienda de la familiar hasta las instalaciones del IDEAUS-CONICET, tomando los recaudos necesarios para garantizar la preservación de los restos durante todo el proceso.

\section{Documentos de Brunet}

Junto con los restos óseos, la familia entregó documentos que formaban parte de la Fundación. Entre ellos se

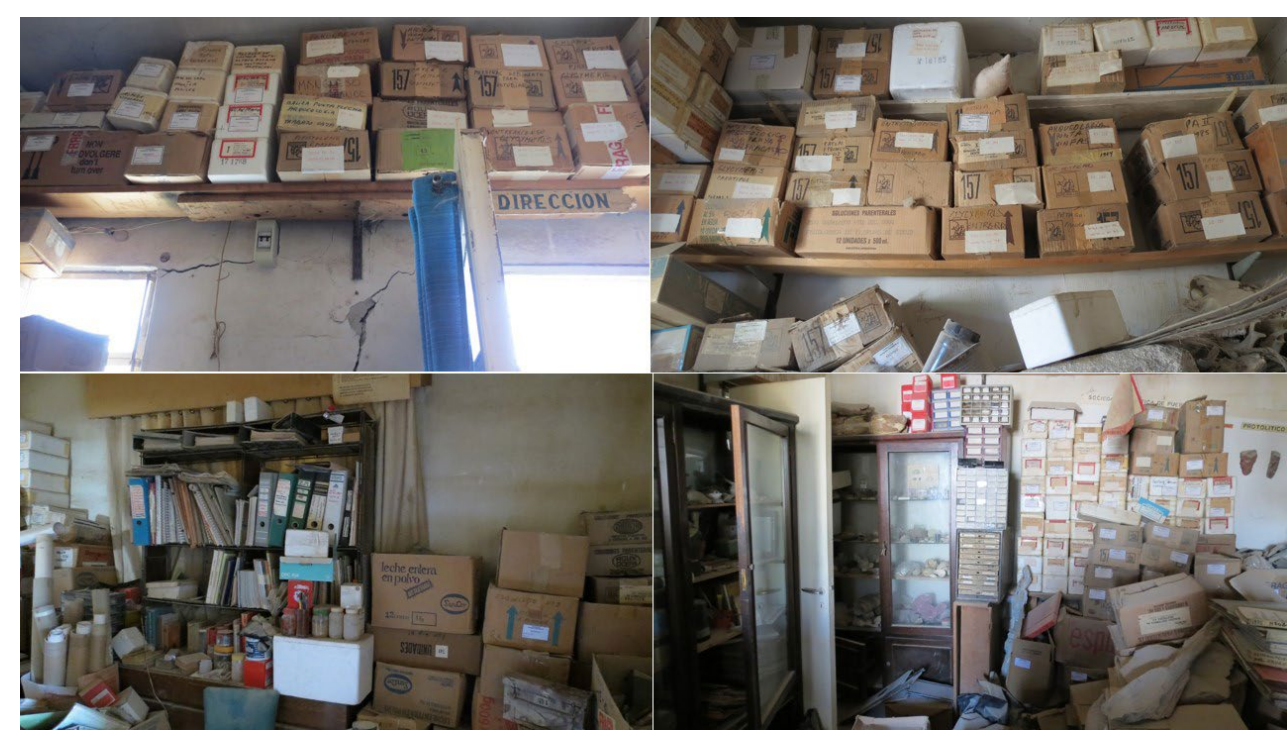

Figura 1. Estado de las colecciones albergadas en el domicilio particular del Sr. Brunet.

Figure 1. Status of the collection housed in $\mathrm{Mr}$. Brunet private home 
encuentra el libro de actas que presenta los números de registro correspondientes a las cajas con materiales y una nota del tipo de material que contiene (por ejemplo: "esqueleto de Puerto Madryn"), sin ninguna otra especificación. Asimismo, se encontraron cuadernos donde señala la ubicación de algunos sitios y esquemas de la posición de hallazgo de los individuos en algunos sitios arqueológicos. Se encontraron también radiografías de algunos elementos óseos, aunque la mayoría no presentan una buena calidad de imagen.

Marco ético y legal de la constitución de la colección Todos los trabajos de conservación siguieron los parámetros éticos señalados por el Código Deontológico para el Estudio, Conservación y Gestión de Restos Humanos de Poblaciones del Pasado de la Asociación de Antropología Biológica Argentina (AABA 2014), que indica que "es responsabilidad de todos los antropólogos biólogos velar por la conservación de restos humanos, practicando y promoviendo su correcta gestión y estudio" y "deben ser tratados con dignidad, sensibilidad y respeto".

La Provincia del Chubut se rige por la Ley Provincial XI No 11 (antes Ley 3559) sobre "Régimen de las ruinas y yacimientos arqueológicos, antropológicos y paleontológicos", que legisla sobre los materiales patrimoniales y establece a la Secretaría de Cultura de Chubut como el ente de aplicación de la mencionada Ley. Dicha Secretaría administra el Registro Único del Patrimonio Arqueológico, Antropológico y Paleontológico, en el que se inscribe todo el material sujeto al régimen de la mencionada Ley y sus tenedores. Asimismo, regula, a través de controles y autorizaciones, la tenencia y el estudio restos arqueológicos.

En marzo de 2018 se promulga la Ley $V^{\circ} N^{\circ} 159$ a partir de la cual la provincia de Chubut adhiere a la Ley Nacional $N^{\circ}$ 25.517. La misma establece en sus tres artículos que los restos óseos de pueblos originarios deben ser puestos a disposición de las comunidades de pertenencia que los reclamen, que las investigaciones científicas deber contar con el consentimiento de las comunidades originarias y en caso de no reclamo pueden continuar en las instituciones que los albergan. Desde hace más de una década el equipo de arqueología y bioantropología del IDEAUS-CONICET trabaja con el aval de las comunidades originarias de región, a través de la Dirección Asuntos Indígenas de la provincia de Chubut y de los representantes del Consejo Provincial de Restitución. En este sentido, la sanción de la Ley Provincial V N 160 - Dto. N 380 "Protocolo de Tratamientos de Restos Humanos Arqueológicos", de 2018 es resultado del trabajo conjunto entre los representantes de las comunidades originarias, los científicos y la Secretaría de Cultura.

\section{Características de la colección}

El análisis bioarqueológico se inició con la determinación del número mínimo de individuos (NMI), del sexo y estimación de edad para los individuos procedentes de un mismo sitio arqueológicos. El sexo fue determinado, sólo en individuos adultos, de acuerdo a las características morfológicas de la pelvis y del cráneo (Buikstra y Ubelaker, 1994) y las características cuantitativas del fémur (Rodríguez Cuenca, 1994; Béguelin y González, 2008).

La edad se estimó en adultos de acuerdo a los métodos propuestos por Todd (1921a, 1921b) y Suchey-Brooks (Brooks y Suchey, 1990) para sínfisis púbica; según la estructura de la superficie auricular (Lovejoy et al. 1985; Meindl y Lovejoy, 1989:140) y a través del método del cierre de las suturas craneales de Masset (1989). Cabe aclarar que el método del cierre de las suturas craneales solo se tuvo en cuenta cuando los individuos no presentaban la pelvis. La edad en subadultos se estimó de acuerdo al desarrollo dentario, la fusión de las epífisis y la longitud de huesos largos utilizando las referencias de Buikstra y Ubelaker (1994) y de Scheuer y Black (2000).

\section{Curación y métodos de denominación de los restos} De acuerdo a Guichen $(1999,2009)$ es necesario diseñar e implementar políticas de conservación preventiva en las colecciones de restos humanos, dado que los mismos son materiales irremplazables, no renovables y son parte fundamental del patrimonio cultural de las poblaciones. Para la conservación a largo plazo de los restos humanos es fundamental adecuar el lugar de trabajo, efectuar una correcta manipulación, clasificar, restaurar, inventariar y documentar el material generando también un bodegaje conveniente (Cronyn, 1990; Sease, 1994; Simmons y Muñoz Saba, 2005; Lemp Urzúa et al. 2008; Aranda y Del Papa, 2009; Aranda et al. 2010; Isaza y Monsalve Vargas, 2011; Aranda et al. 2016).

Así, en el IDEAUS-CONICET los espacios de trabajo (laboratorio y reservorio) son adecuados para la conservación a largo plazo de las colecciones de restos humanos. El laboratorio es amplio y luminoso, cuenta con mesadas firmes y de tamaño conveniente para la manipulación de los materiales. El reservorio es un lugar destinado al bodegaje de diferentes colecciones biológicas que se encuentra a temperatura y humedad constante durante todo el año y se halla libre de plagas, cuenta además con estanterías metálicas firmes donde se depositan las cajas con el material biológico. Los elementos óseos de cada esqueleto fueron embolsados, guardados en cajas nuevas y finalmente ubicadas en dichas estanterías (Figura 2), manteniendo las barreras de aislamiento apropiadas para garantizar la protección de los materiales.

Quedó entonces establecida formalmente la "Colección Bioantropológica Frengüelli-Brunet" bajo custodia del IDEAUS-CONICET, teniendo como curadora a una de las autoras (SD). 


\section{Resultados}

La colección se integra por un número mínimo de 67 individuos, de los cuales 56 proceden de la provincia de Chubut (Figura 3), uno de San Antonio Oeste, provincia de Río Negro y 10 no cuentan con procedencia. De los

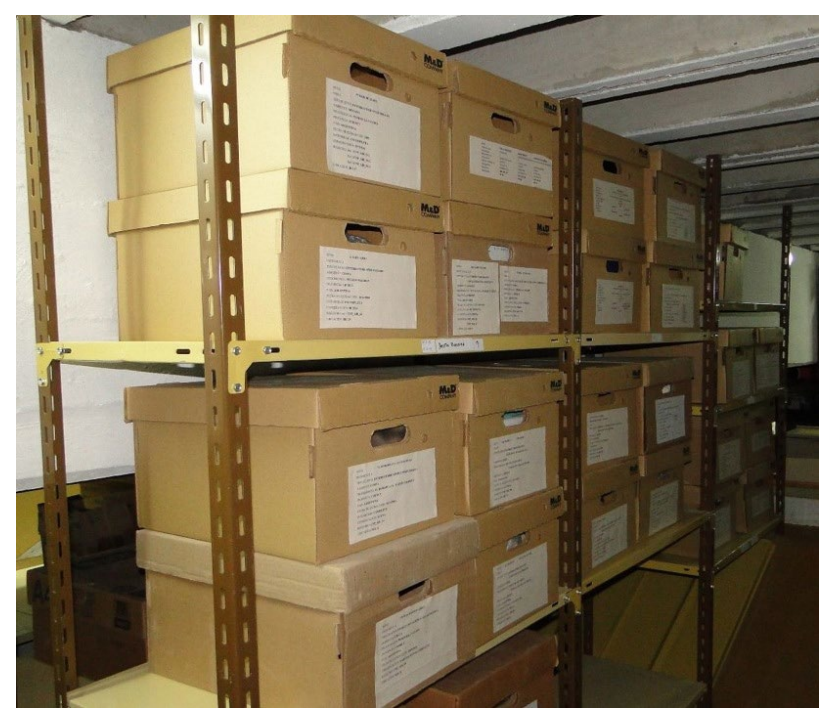

Figura 2. Colección Bioantropológica Frengüelli-Brunet en las instalaciones del IDEAUS-CONICET.

Figure 2. Bioanthropological Frengüelli-Brunet collection located in IDEAUS-CONICET building.
56 individuos recolectados en Chubut 33 provienen del Departamento Florentino Ameghino de los sitios Cabo Dos Bahías, Camarones, Punta Rojas, Punta Atlas y Punta Tombo. Del Departamento Biedma 10 individuos, de los sitios Puerto Madryn, El Indio (Puerto Madryn), el Riacho y Punta Norte. Del Departamento Paso de Indios, proviene un individuo del sitio Cerro Cóndor. Del Departamento Tehuelches, sitio Laguna Blanca, provienen tres individuos. Del Departamento Languiñeo, sitio Estancia La Mimosa, proceden nueve individuos. En cuanto a la representatividad geográfica de los esqueletos humanos de Colección mayoritariamente proceden de la costa de Chubut; costa noreste (11 individuos) y la costa central (33 individuos); la minoría son de la región centrooeste (trece individuos) (Figura 3).

Del total de 67 individuos se identificaron 42 esqueletos individualizados (10 femeninos, 16 masculinos y 16 de sexo indeterminado, 36 adultos y seis subadultos) y siete conjuntos de restos mezclados con mínimo de 25 individuos. (Tabla 1).

Del total de individuos 12 son subadultos (18\%) y 55 adultos (82\%). En cuanto a la determinación del sexo, de los 55 adultos, 12 son femeninos (22\%), 22 son masculinos (40\%) y 21 (38\%) son individuos de sexo indeterminado (Tabla 1). En cuanto a la estimación de la edad, de los 55 adultos seis son adultos jóvenes (11\%), siete son adultos medios (13\%), tres adultos mayores (5\%) y los 39 restantes (71\%) son adultos que deben ser

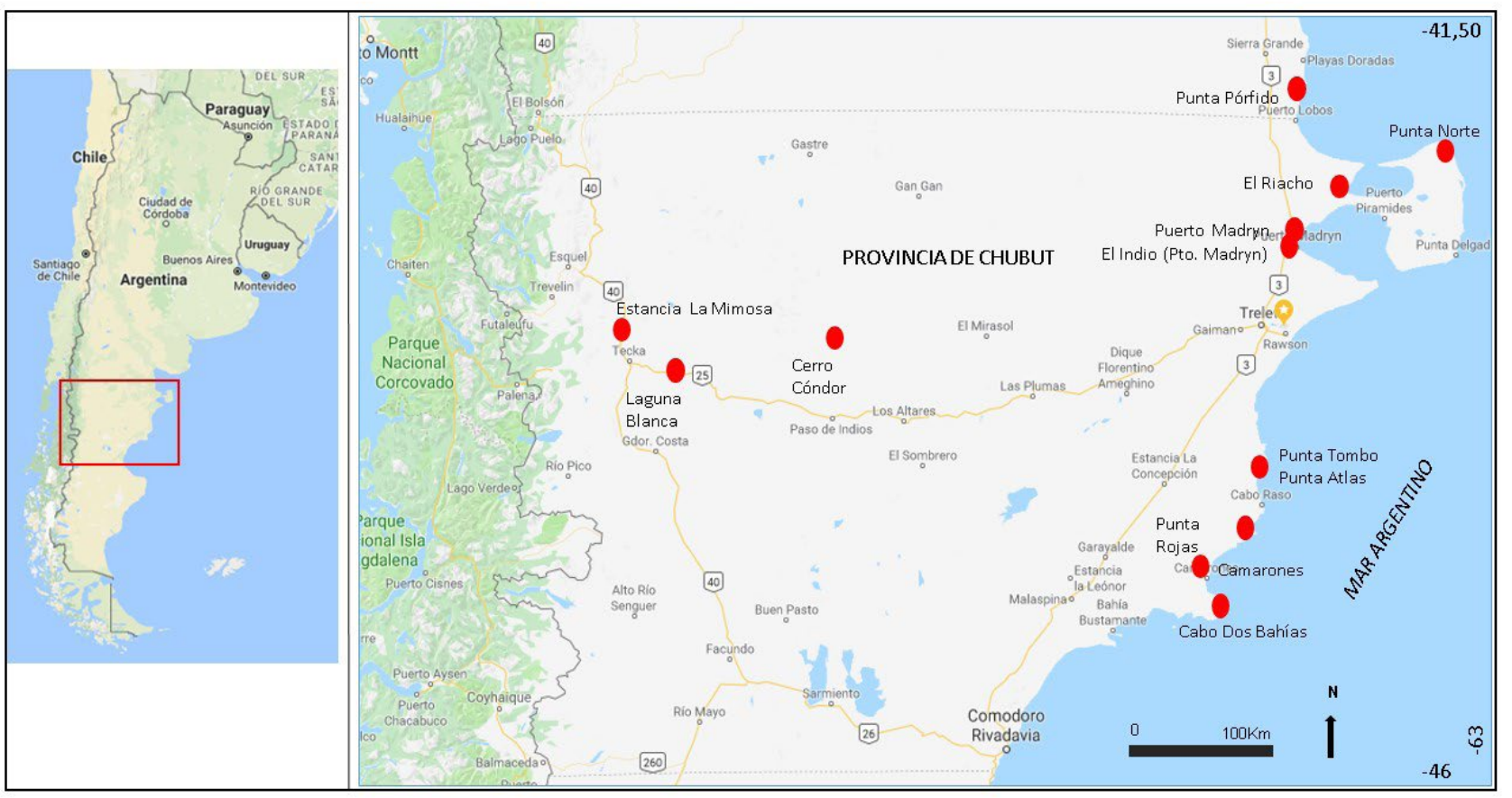

Figura 3. Procedencia de los individuos que conforman la Colección Frengüelli-Brunet.

Figure 3. Geographical origin of individuals that make up Frengüelli-Brunet collection 
Tabla 1. Restos óseos que integran la colección Frengüelli-Brunet.

Table 1. Bone remains in Frengüelli-Brunet collection

\begin{tabular}{|c|c|c|c|c|c|}
\hline Provincia & Departamento & Sitio & NMI & Sexo & Edad \\
\hline \multirow{49}{*}{ Chubut } & Florentino Ameg. & Cabo 2 Bahías - Ind.1 & 1 & $\mathrm{M}$ & Adulto Medio (35-50 años) \\
\hline & Florentino Ameg. & Cabo 2 Bahías - Ind.2 & 1 & 1 & Sub Adulto (2-4años) \\
\hline & Florentino Ameg. & Cabo 2 Bahías - Ind.3 & 1 & $\mathrm{~F}$ & Adulto Medio (30-40 años) \\
\hline & Florentino Ameg. & Cabo 2 Bahías - Ind.4 & 1 & $\mathrm{M}$ & Adulto Medio (35-50 años) \\
\hline & Florentino Ameg. & Cabo 2 Bahías - Ind.5 & 1 & $\mathrm{M}$ & AdultoMayor(>50años) \\
\hline & Florentino Ameg. & Cabo 2 Bahías - Ind. 6 & 1 & $\mathrm{M}$ & Adulto Joven (18-25 años) \\
\hline & Florentino Ameg. & Cabo 2 Bahías - R. Mezc. & 2 & 1 & Adultos \\
\hline & Florentino Ameg. & Camarones - R. Mezc. & 2 & $\mathrm{M}$ & Adultos \\
\hline & Florentino Ameg. & Camarones - Ind.1 & 1 & $\mathrm{~F}$ & Adulto Joven (20-30 años) \\
\hline & Florentino Ameg. & Camarones - Ind.2 & 1 & 1 & Sub Adulto (16-17años) \\
\hline & Florentino Ameg. & Punta Rojas - Ind.1 & 1 & $M$ & Adulto Medio (35-50 años) \\
\hline & Florentino Ameg. & Punta Rojas - Ind.2 & 1 & $M$ & Adulto \\
\hline & Florentino Ameg. & Punta Rojas - Ind.3 & 1 & 1 & Adulto \\
\hline & Florentino Ameg. & Punta Rojas - Ind.4 & 1 & 1 & Adulto \\
\hline & Florentino Ameg. & Punta Rojas - Ind.5 & 1 & F? & Adulto \\
\hline & Florentino Ameg. & Punta Rojas - Ind.6 & 1 & $M$ & Adulto \\
\hline & Florentino Ameg. & Punta Rojas - Ind.7 & 1 & 1 & Sub adulto (4-6años) \\
\hline & Florentino Ameg. & Punta Rojas - Ind.8 & 1 & $\mathrm{~F}$ & Adulto Medio (30-45 años) \\
\hline & Florentino Ameg. & Punta Rojas - Ind.9 & 1 & $M$ & Adulto Medio (35-50 años) \\
\hline & \multirow[t]{4}{*}{ Florentino Ameg. } & \multirow[t]{4}{*}{ Punta Rojas - R. Mezc. } & 2 & 1 & Adultos \\
\hline & & & 1 & 1 & Sub adulto \\
\hline & & & 1 & $\mathrm{~F}$ & Adulto Medio (30-40 años) \\
\hline & & & 1 & $M$ & Adulto Mayor (40-50 años) \\
\hline & \multirow[t]{5}{*}{ Florentino Ameg. } & \multirow[t]{5}{*}{ Punta Atlas - R. Mezc. } & 1 & $\mathrm{M}$ & Adulto Joven (20-35 años) \\
\hline & & & 1 & $\mathrm{M}$ & Adulto \\
\hline & & & 1 & $\mathrm{~F}$ & Adulto Joven (20-30 años) \\
\hline & & & 1 & 1 & Adulto \\
\hline & & & 1 & 1 & Sub Adulto \\
\hline & Florentino Ameg. & Punta Tombo - Ind.1 & 1 & 1 & Adulto \\
\hline & Florentino Ameg. & Punta Tombo - Ind. 3 & 1 & 1 & Adulto \\
\hline & Biedma & Puerto Madryn - Ind.1 & 1 & $\mathrm{M}$ & Adulto \\
\hline & Biedma & Puerto Madryn - Ind.3 & 1 & 1 & Adulto \\
\hline & Biedma & Puerto Madryn - Ind.4 & 1 & 1 & Sub Sdulto \\
\hline & Biedma & Puerto Madryn - Comisaría & 1 & $\mathrm{~F}$ & Adulto \\
\hline & \multirow[t]{2}{*}{ Biedma } & \multirow{2}{*}{$\begin{array}{c}\text { El Indio-Puerto Madryn } \\
\text { - R. Mezc. }\end{array}$} & 1 & 1 & Adulto \\
\hline & & & 1 & 1 & Sub Adulto \\
\hline & Biedma & El Riacho - Ind.1 & 1 & $M$ & Adulto \\
\hline & \multirow[t]{2}{*}{ Biedma } & \multirow[t]{2}{*}{ Punta Norte - R. Mezc. } & 2 & $\mathrm{I}$ & Adultos \\
\hline & & & 1 & 1 & Sub Adulto \\
\hline & Paso de Indios & Cerro Cóndor - Ind.1 & 1 & 1 & Adulto \\
\hline & Tehuelches & Laguna Blanca - Ind.1 & 1 & 1 & Adulto \\
\hline & Tehuelches & Laguna Blanca - Ind.2 & 1 & $\mathrm{~F}$ & Adulto \\
\hline & Tehuelches & Laguna Blanca - Ind. 3 & 1 & $\mathrm{M}$ & Adulto Joven \\
\hline & Languiñeo & Estancia La mimosa - Ind.1 & 1 & $M$ & Adulto \\
\hline & Languiñeo & Estancia La mimosa - Ind.2 & 1 & $\mathrm{~F}$ & Adulto \\
\hline & Languiñeo & Estancia La mimosa - Ind.3 & 1 & 1 & Adulto \\
\hline & \multirow[t]{3}{*}{ Languiñeo } & \multirow{3}{*}{$\begin{array}{c}\text { Estancia La mimosa } \\
\text { - R. Mezc. }\end{array}$} & 3 & 1 & Adultos \\
\hline & & & 1 & $M$ & Adulto \\
\hline & & & 2 & 1 & Sub Adultos \\
\hline Río Negro & Sierra Grande & P. Pórfido - Ind.1 & 1 & $\mathrm{~F}$ & Adulto \\
\hline \multirow{10}{*}{ Se desconoce } & Se desconoce & \#11 (Cráneo y mandibula) & 1 & $M ?$ & Adulto Joven \\
\hline & Se desconoce & \#22 ( J-113) (Cráneo y mandibula) & 1 & $\mathrm{M}$ & Adulto \\
\hline & Se desconoce & \#4 Cráneo y mandibula 4 & 1 & 1 & Sub adulto \\
\hline & Se desconoce & \#3 (Fragmento calota) & 1 & 1 & Adulto \\
\hline & Se desconoce & \#20 (I 134) ( Cráneo) & 1 & $\mathrm{~F}$ & Adulto \\
\hline & Se desconoce & \#21 ( Cráneo) & 1 & $\mathrm{M}$ & Adulto Mayor \\
\hline & Se desconoce & \#13 ( Cráneo y mandibula) & 1 & $\mathrm{~F}$ & Adulto \\
\hline & Se desconoce & NN1 (cráneo y mandíbula) & 1 & $M ?$ & Adulto \\
\hline & Se desconoce & NN2 (Fragmento calota) & 1 & $\mathrm{I}$ & Adulto \\
\hline & Se desconoce & NN3 (Fragmento calota) & 1 & 1 & Sub adulto \\
\hline \multicolumn{3}{|r|}{ TOTAL } & 67 & & \\
\hline
\end{tabular}


analizados en profundidad o que no es posible asignarle una categoría etaria.

\section{Discusión}

Las colecciones de restos humanos contribuyen al fortalecimiento de la identidad y ofrecen herramientas importantes en el ámbito científico para reconstruir los estilos de vida de las sociedades del pasado. Es por ello, que se hace necesario no sólo incluir en su custodia aspectos técnicos de conservación, sino también establecer políticas de manejo, documentación y sistematización de la información (Aranda y Del Papa, 2009; Aranda et al. 2010; Lemp Urzúa et al. 2008; Millán y Dahinten, 2019).

Las colecciones, como las define Pomian (1990), "son instituciones coextensivas al hombre en el tiempo y en el espacio, cuyo principal papel es el vínculo entre lo visible y lo invisible". "Hay una historia del comportamiento que acompaña a la creación de las colecciones sea cual fuere el objeto a coleccionar". En el caso de las colecciones biológicas, al morir los coleccionistas, muchas piezas son trasladadas a museos o centros científicos, donde el rol fundamental de estas instituciones ha sido la conservación y protección para favorecer su persistencia a través del tiempo. Las colecciones de restos humanos no han sido la excepción, y en este sentido la colección FrengüelliBrunet responde a estas características.

En contraposición a la forma en que este conjunto de restos humanos fue conformado, el IDEAUS-CONICET alberga la colección institucional, que tiene sus orígenes en la década del '90 a partir de rescates arqueológicos dirigidos por la Dra. Julieta Gómez Otero. Se conforma por restos óseos humanos procedentes en su mayoría de la Provincia de Chubut y se encuentra bajo la curaduría de la Dra. Silvia Dahinten (Gómez Otero y Dahinten 1997/98; Millán y Dahinten 2019; Millán, Gómez Otero y Dahinten, 2013). Por el contrario, los restos de la Colección Frengüelli-Brunet fueron recuperados en gran parte de la costa central, en las proximidades de la Bahía Camarones. Por tal motivo, su acondicionamiento, resguardo y estudio permite no solo mejorar sus condiciones de conservación bajo marcos éticos-legales, sino también ofrece una oportunidad significativa para aportar información bioantropológica sobre las poblaciones del pasado de esta particular región, para la cual hasta el momento no existían restos óseos disponibles para estudio.

En la actualidad la recuperación de material de sitios arqueológicos en la provincia de Chubut, se encuentra reglamentada por la Ley Provincial $\mathrm{V} \mathrm{N}^{\circ} 160$. La misma establece normas y pautas relacionadas al protocolo de tratamiento de restos humanos arqueológicos: el hallazgo, la verificación in situ y el trabajo de campo deben contar con la presencia de veedores referentes de zona. A pesar de haber sido conformada por fuera de la legislación correspondiente y de carecer de los controles arqueológicos necesarios, la Colección Frengüelli-Brunet puede ser tomada como una fuente de información posible, limitada en algunos casos por la ausencia de datos de contexto arqueológicos.

En la actualidad la colección Frengüelli-Brunet transita las primeras etapas de curación y almacenamiento. Aún resta analizar en detalle algunos sitios, se están profundizando los análisis de determinación de edad con el fin de lograr una mayor precisión en su estimación, se evalúa también que puedan encontrarse nuevos restos humanos lo que ampliaría los resultados presentados en este trabajo. Paralelamente se están realizando estudios complementarios de datación radiocarbónica, de isótopos estables y moleculares de ADN. El avance de los estudios de la colección permitirá ampliar la información ya obtenida para poblaciones de cazadores recolectores de la costa de Chubut.

\section{Conclusiones}

Los restos humanos de la Colección Frengüelli-Brunet incluyen un mínimo de 67 individuos, la mayor parte de ellos adultos. Las tareas realizadas en la colección, además de poner en valor y resguardo los de restos recuperados en forma privada y sin controles arqueológicos, permiten incrementar la información disponible para la costa central y la región centro oeste de Chubut región aún con escasos datos bioantropológicos. Trabajos futuros permitirán analizar estos individuos conjuntamente con otros restos que posean datos contextuales, fortaleciendo así la información regional.

Puerto Madryn, 2 de Mayo 2019

\section{Agradecimientos}

Las autoras agradecen a la Secretaría de Cultura de la Provincia del Chubut, en especial a la Prof. Evelyn Beroiza por sus gestiones y su activa participación durante el traslado de los restos óseos humanos a las instalaciones del IDEAUS-CONICET. Agradecen especialmente a la Dra. Julieta Gómez Otero, quien fue el nexo entre los familiares de Rodolfo Brunet y a su equipo quienes trabajaron durante el traslado. También agradecen al Dr. Jorge Suby, por sus valiosos comentarios y aportes en el presente trabajo y a la Lic. Camila Tamburrini por la ayuda proporcionada en la traducción al inglés del resumen.

\section{Referencias Bibliográficas}

AABA. 2014. Código deontológico para el estudio, conservación y gestión de restos humanos de poblaciones del pasado. Redactado por C. Aranda, G. Barrientos y M. Del Papa (Sub-Comisión de Ética de la Asociación de 
Antropología Biológica Argentina). Revista Argentina de Antropología Biológica, 16(2), 111-113.

Aranda, C. y M. Del Papa. 2009. Avances en las prácticas de conservación y manejo de restos humanos en argentina. Revista Argentina de Antropología Biológica, 11(1),89-93.

Aranda, C., K. Zuccala, D. Avido, A. Salvarredy, L. Luna y V. Gigliotti. 2010. Manejo de colecciones osteológicas del museo etnográfico J.B. Ambrosetti (FFyL, UBA). Recuperado de http://www.researchgate. net/publication/256458348.

Aranda, C., L. Luna, N. Costanzo, V. Contissa, N. Gámez, P. Godoy y K. Zuccala. 2016. Conservación e investigación en el área de Antropología Biológica del Museo Etnográfico J. B. Ambrosetti (Facultad de Filosofía y Letras, Universidad de Buenos Aires, Argentina) DOI: http://dx.doi.org/10.14195/2182-7982_31_1

Béguelin, M y P. N. González. 2008. Estimación del sexo en poblaciones del sur de Sudamérica mediante funciones discriminantes para el fémur. Revista Argentina de Antropología Biológica, 10(2), 55-70.

Brooks, S. y J. M. Suchey. 1990. Skeletal age determination based on the os pubis: a comparison of the AcsadiNemeskeri and Suchey broks methods. American Journal of Physical Anthropology, 3, 227-238.

Brunet, Rodolfo F. J. 1983. Manual de malacología. Cómo coleccionar conchillas. Impresora Golfo Nuevo. Chubut, Argentina.

Brunet, Rodolfo F. J. 1995. Manual de paleontología. Unicamente fósiles de la Argentina. Buenos Aires, Argentina: Cumacú.

Buikstra, J. E. y D. H. Ubelaker. 1994. Standards. For data collection from human skeletal remains. Arkansas Archeological Survey Research Series $N^{\circ} 44$. Arkansas.

Cronyn, J. M. 1990. The elements of archaeological conservation. London: TJ Press.

Gómez Otero, J. y S. L. Dahinten. 1997/98. Costumbres funerarias y esqueletos humanos: variabilidad y poblamiento en la costa nordeste de la provincia del Chubut. Relaciones de la Sociedad Argentina de Antropología, XXII-XXIII, 101-124.

Guichen, G. 1999. La conservación preventiva: ¿Simple moda pasajera o cambio transcendental? En Museo Internacional. 201 (1) Paris.

Guichen, G. 2009. Medio siglo de Conservación Preventiva. Entrevista a Gael de Guichen. www.revista. ge-iic.com. Ge-conservación nº 0 .

Isaza, J. y T. Monsalve Vargas. 2011. Características biológicas de la colección osteológica de referencia de la Universidad de Antioquia. Informe preliminar. En http:// www.researchgate.net/publication/236671022.

Lemp Urzua, C., M. Rodríguez Balboa, R. Retamal Yermani y E. Aspillaga Lafontaine. 2008. Arqueología del depósito: manejo integral de las colecciones bioantropológicas en el Departamento de Antropología de la Universidad de Chile. Conserva Nro 12.

Ley $\mathrm{V} \mathrm{N}^{\circ} 160$-Dto. N 380. Protocolo de Tratamientos de Restos Humanos Arqueológicos. Boletín Oficial Año LX - N 12946. Provincia de Chubut.

Ley V No 159 - Dto. No 139 - Adhesión a la Ley Nacional $N^{\circ} 25.517$ de Restitución de Restos Mortales de Aborígenes. Boletín Oficial Año LX - Nº 12884. Provincia de Chubut

Ley XI N 11(Antes Ley 3559). Digesto Jurídico, Provincia del Chubut. Extraído de http://www.legischubut2.gov.ar/ digesto/|xI/XI-11.html

Ley Nacional N²5.517. Restitución de Restos Mortales de Aborígenes.

Lovejoy, C., R. S. Meindl, T. R. Prizbeck, y R. Mensforthr. 1985. Chronological metamorphosis of the auricular surface of the ilium: a new method for the determination of adult skeletal age at death. American Journal of Physical Anthropology, 68, 15-28.

Masset, C. 1989. Age Estimation on the Basis of Cranial Sutures. In Age Markers in the Humann Skeleton, edited by M. Y. Iscan, 71-103. Charles C. Thomas, Springfield, Illinois.

Meindl, R. S. y C. O. Lovejoy. 1985. Ectocranial Suture Closure: A Revised Method for the Determination of Skeletal Age at Death Based on the Lateral-Anterior Sutures. American Journal of Physical Anthropology, $68,57-66$.

Millán, A. G, J. Gómez Otero y S. Dahinten. 2013. Tendencia secular de la estatura en poblaciones humanas del valle inferior del rio Chubut y de la costa centro-septentrional (Patagonia Argentina) durante el holoceno tardío. Relaciones de la Sociedad Argentina de Antropología, XXXVIII (2), 421-440.

Millán, A. G. y S. Dahinten. 2019. La Colección Bioantropológica del norte de Chubut (Secretaría de Cultura de Chubut / IDEAUS-CONICET): Integración, conservación e investigación. En Arqueología de la Patagonia: El pasado en las arenas, 205-213. Buenos 
Aires, Argentina.

Pomian, K. 1990. Collectors and Curiosities: Paris and Vienna, 1500-1800. Cambridge: Polity Press.

Rodríguez Cuenca., J. V. 1994. Introducción a la Antropología Forense, Análisis e identificación de restos óseos humanos. Santafé de Bogotá, Colombia: Anaconda Editores

Scheuer, L. y S. Black. 2000. Developmental Juvenile Osteology. California: Elsevier Academic Press.

Sease, C. 1994. A conservation manual for the field Archaeologist. Third edition. Archaeological Reserch
Tools, Volume 4. of Archaeology Institute. University of California, Los Angeles.

Simmons, J. E. y Y. Muñoz Saba. 2005. Cuidado, manejo y conservación de las Colecciones Biológicas. Universidad Nacional de Colombia.

Todd, T. 1921 a. Age Changes in the Pubic Bone. I: The Male White Pubis. American Journal of Physical Anthropology 3:285-334.

Todd, T. 1921 b. Age Changes in the Pubic Bone. III: The Pubis of the White Female. IV: The pubis of the female white-negro hybrid. American Journal of Physical Anthropology, 4:1-70. 